\title{
Research on standardized training of resident doctors based on competence
}

\author{
Ma Huining', Li Feng², Liang Yuqing², Li Zhongyuan', Sun Maolin' \\ ${ }^{1}$ Qiqihar Medical College Affiliated First Hospital, Qiqihar, China, 161041, \\ ${ }^{2}$ Qiqihar Medical University, Qiqihar, China, 161006
}

Keywords: competency; resident physician; standardized training; quality management; assessment system

Abstract: As an important part of post-graduate medical education, standardized training for residents plays an indispensable role in the good professional ethics of residents, solid medical theoretical knowledge and clinical skills. How to ensure the quality of training and improve the training effect is an urgent problem to be solved in the work. Combining with the practice of standardized training for doctors in our hospital, we will explore the construction of a standardized training and evaluation system for residents, and provide practical and reasonable suggestions for improving the quality of standardized training for residents.

\section{Introduction}

In August 2014, the National Health and Family Planning Commission and the Chinese Medical Doctor Association jointly carried out the certification of resident standardized training bases nationwide. On August 25, 2014, the "Regulations on the Management of Resident Standardized Training (Trial)" was issued, marking the resident of China. Standardized training work has entered new milestones. However, how to effectively carry out standardized training for residents, ensure the quality of standardized training for residents, and establish a perfect standardized training and evaluation system for residents has become an indispensable focus. In view of the fact that the strategy of the evaluation system used by the hospital during the training process plays an important role in ensuring the quality of the training, since the implementation of the standardized training for residents in 2010, the Institute has continuously explored the practice of the standardized training and assessment system for residents and continued to improve.

\section{The main content of the resident training standardized assessment system}

Constructing a standardized training and evaluation system for residents is not only for the assessment of residents, but for the assessment of standardized training for the entire resident. At the same time, the management of the training is included in the annual comprehensive target assessment of the department, including the assessment of residents and residents. The assessment content of Dr. Pei includes daily management assessment, outpatient assessment and annual theoretical skills assessment. The year-end assessment with the teacher includes the admission 
management of the doctor, the development of the training plan, the implementation of the training plan and the evaluation of the physician.

\section{Daily management assessment of resident standardized training}

\subsection{Public Course Training and Assessment}

Physicians must participate in public course training and special training organized by the Resident Standardized Training Management Office (hereinafter referred to as the Regulation and Training Office). In addition to training medical related laws and regulations, in-house rules and regulations, and related medical and therapeutic techniques, public course training also includes lectures on humanities knowledge, professional ethics, and communication skills between doctors and patients. While training the clinical literacy of physicians, it pays attention to the cultivation of humanistic literacy, professional ethics, teamwork ability, and communication ability between doctors and patients. For special reasons, you should take a formal leave request and report to the office.
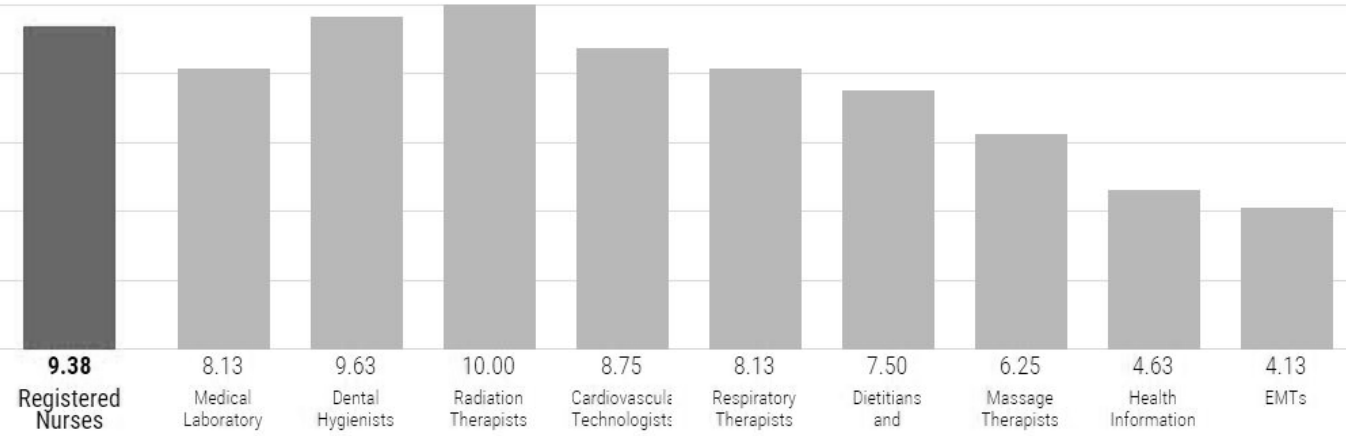

Fig.1 Statistics on the assessment of resident public course training

\subsection{Medical record writing quality assessment}

A complete and standardized medical record is the basic method to cultivate the clinical thinking ability of clinicians, and is an important way to improve the clinical level of clinicians. The doctors who rotate in each department must write their own work number and complete the corresponding medical records. The regulatory office regularly checks the running medical records. For the medical records of the doctors, the quality of the medical records includes the evaluation of "quality" and the "quantity". For the examination of the "quality" of the medical records, the mode of random inspection can be selected, and the records of the first pass and the records of the residents' rounds should be checked. The content written by the physician, the time limit for writing the medical record and the quality of the written content, etc., are not the responsibility of the resident to all the problems in the medical record. The examination content of the "quantity" of running medical records is the number of medical records completed by each month through the statistics of the physicians, and the number of written records of the medical records of different rotation departments is qualified, otherwise it is unqualified.

\subsection{On-the-job supervision}

What has to be said is that many hospitals have the phenomenon that medical graduates are assigned to hospitals to fix departments. Due to the adjustment of the system and the expansion of bed size, the contradiction between supply and demand of some departments is prominent, which 
causes some departments to require the rotation of the staff to participate in the work. . The sufficient rotation training room training time is a necessary condition for ensuring the effectiveness of resident standardized training. The Regulation and Training Office conducts monthly supervision on the on-the-job physician's on-the-job status, and finds that the doctor has not performed the leave process and is not in the post. Relevant departments, increase penalties, and fail to qualify for the annual assessment.

\section{Implementation assessment of resident standardized training program}

\subsection{Assessment of teaching rounds}

The ward round is a core component of medical work, with a focus on completing clinical diagnosis and treatment tasks. Compared with the clinical ward round, the fundamental of clinical teaching ward round is to improve the comprehensive diagnosis and treatment level of lower level doctors through the standardized process, and complete the corresponding clinical tasks. Teachers who teach can't be "trained by the workers". In addition to teaching the doctors in the daily rounds, they also need to organize clinical teaching rounds regularly, no less than 2 times a month.

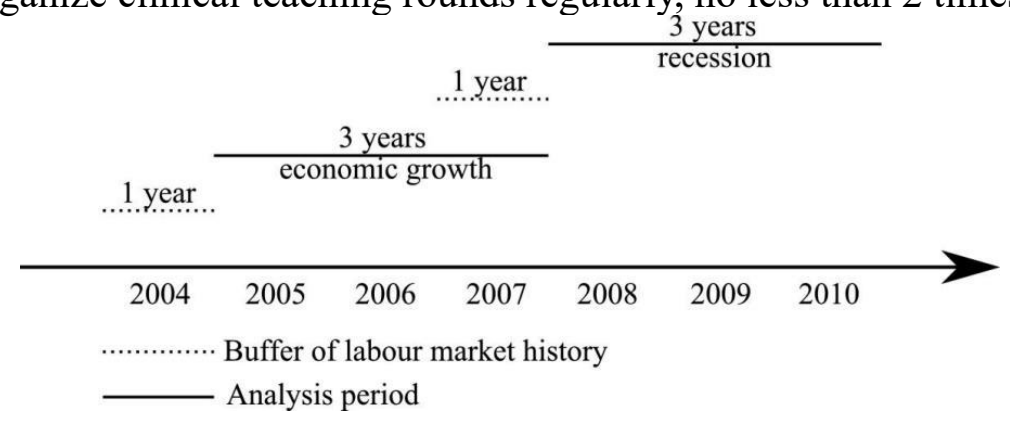

Fig.2 Revenue trends of resident standardized training

\subsection{Assessment of difficult case analysis and discussion}

The transfer department needs to organize and analyze the difficult cases every month. The teachers and the doctors in the department need to participate and discuss in stages. The teacher with the teacher needs to register the patient's name, hospitalization number, diagnosis and specific discussion time, and the doctors involved in the discussion to the teaching manual. The regulatory office regularly supervises and does not complete the itemized deduction.

\subsection{Assessment of lectures within the department}

The transfer department needs to organize monthly lectures in the department, and the teachers in the department take turns to give lectures. The contents of the lectures can focus on the routine interpretation of the diagnosis and treatment of the disease, the latest clinical treatment progress of the disease, and the communication skills of doctors and patients. The teacher will register the specific lecture title, time and participants to the teaching manual and save the lecture report PPT and lecture photos for future reference.

\section{Advantages of resident standardized training and evaluation system}

\subsection{Competency-oriented}

The core of the assessment system is competency-oriented. Competency is generally defined as 
the integration of all capabilities that must be learned in a particular position. In order from lowest to highest, competency can often be divided into motivation, traits, self-concept (including self-image, attitude or values), knowledge and skills.

$$
\text { Score }=\mathrm{KPI}+\mathrm{KCI}
$$

(Assessment Indicator $=$ Key Performance Indicator + Key Competency Indicator)

The Physician passed the resident standardization training and completed the examination, and obtained the certificate of completion of the resident medical training, so that the requirements for promotion in the future are the goal of the standardized training of the resident rather than the ultimate goal. The core of the competency-oriented training and appraisal physicians as the standardized training and evaluation system for residents is to cultivate professional, communication, collaboration, management, health promotion and professional ethics to face patients independently. Therefore, the resident doctor's theory and skill assessment accounted for 30\% of the entire assessment system.

\subsection{Combination of doctors and teachers with teachers}

The combination of disciplined physicians and teachers with assessment is one of the important breakthroughs in the assessment system. The traditional assessment mode pays more attention to the management and assessment of the doctors. It is understandable, but it is more dependent on the high-quality management and teaching team. There may be a phenomenon of not being active, using and training lightly. The assessment system constructed in this paper, at the same time, assesses the resident doctors and teachers.

\section{Summary}

As an important part of post-graduate medical education, resident standardized training plays an indispensable role for the resident's good professional ethics, solid medical theoretical knowledge and clinical skills. How to ensure the quality of training and improve the training effect is an urgent problem to be solved in the work. How to effectively carry out standardized training for residents, ensure the quality of standardized training for residents, and establish a perfect standardized training and evaluation system for residents has become an indispensable focus.

\section{Acknowledgement}

This project is support by The 13th Plan of Higher Education Research of the Higher Education Institute of Heilongjiang Province "Study on the construction of a standardized training teacher team for residents centered on trainees"(16G280)

\section{References}

[1] Chen Y, Zhang Y P, Ma J. Establishment of competency model after resident doctor standardization training[J]. Journal of Shanghai Jiaotong University, 2014, 43(5):1173-1178.

[2] Zhou J. The study on core competence of the resident doctors in the resident standardization training bases[J]. Chinese Health Resources, 2015.

[3] Luo L Z, Pan H, Zhang Y, et al. Discussion on the competency training of the management staff in the resident standardized training base[J]. Basic \& Clinical Medicine, 2017. 